\title{
Non-material Finite Element Modelling of Large Vibrations of Axially Moving Strings and Beams
}

\author{
Yury Vetyukov \\ Vienna University of Technology, Institute of Mechanics and Mechatronics, \\ Getreidemarkt 9, A-1060 Vienna, Austria
}

\begin{abstract}
We present a new mathematical model for the dynamics of a beam or a string, which moves in a given axial direction across a particular domain. Large inplane vibrations are coupled with the gross axial motion, and a Lagrangian (material) form of the equations of structural mechanics becomes inefficient. The proposed mixed Eulerian-Lagrangian description features mechanical fields as functions of a spatial coordinate in the axial direction. The material travels across a finite element mesh, and the boundary conditions are applied in fixed nodes. Beginning with the variational equation of virtual work in its material form, we analytically derive the Lagrange's equations of motion of the second kind for the considered case of a discretized non-material control domain and for geometrically exact kinematics. The dynamic analysis is straightforward as soon as the strain and the kinetic energies of the control domain are available. In numerical simulations we demonstrate the rapid mesh convergence of the model, the effect of the bending stiffness and the dynamic instability when the axial velocity gets high. We also show correspondence to the results of fully Lagrangian benchmark solutions.
\end{abstract}

Keywords: axially moving structures, spatial description, beams and strings, large vibrations, nonlinear finite element modelling, time integration 2010 MSC: 35Q74, 74A05, 74H15, 74K05, 74K10, 74S05

\section{Introduction}

Axially moving strings or beams have long attracted the attention of mechanical engineers. Practical relevance, technical difficulties in maintaining the desired regime of motion, non-trivial and even sometimes counter-intuitive behavior are coupled with challenges, intrinsic for the theoretical investigation of such systems. Corresponding mathematical models traditionally feature a spatial (or Eulerian) description, see review papers by Chen [1] and Marynowski and

Email address: yury.vetyukov@tuwien.ac.at (Yury Vetyukov)

(C) 2018. This is the peer reviewed version of the following article: Vetyukov, Y. (2018). Non-material finite element modelling of large vibrations of axially moving strings and beams. Journal of Sound and Vibration, 414, 299-317, which has been published in final form at https://doi.org/10.1016/j.jsv.2017.11.010. This manuscript version is made available under the CC-BY-NC-ND 4.0 license http://creativecommons.org/ licenses/by-nc-nd/4.0/ 
Kapitaniak [2]. Considering unknowns displacements, forces, moments, etc. as functions of a fixed coordinate in the axial direction simplifies the analysis, as the problem needs then to be solved in a fixed control domain and the boundary conditions are applied at fixed points. On the other hand, the basic equations of structural mechanics are available in the Lagrangian form, when the mechanical fields are observed in material points. As shown below in section 2 , transforming the equations to the spatial form is simple in the linear case, when the 15 relation between the material and the spatial coordinates is known in advance. In nonlinear problems, many authors adopt certain kinematical simplifications and derive the mathematical model in the spatial frame from scratch [3, 4, or rely upon other known equations from the literature. Thus, the models of Wickert [5] and Mote [6] are often applied, see e.g. [7, 8, 9, 10. While numerous

20 fascinating and practically important results were obtained in this way, accurate modelling of large vibrations of axially moving structures requires exact treatment of geometrically nonlinear effects both in the elastic response as well as in the inertial properties with an established structural mechanics theory in the background. Despite growing interest of researchers [11, 12, 13, 14, 15, 16], de25 veloping efficient and reliable techniques of transforming the general equations of motion to a new spatial form remains a challenging problem.

In computational mechanics of flexible solids, dynamical modelling of a discretized system is convenient with Lagrange's equations of motion of the second kind. For a given finite element (or global Ritz) approximation one simply 30 integrates the total strain energy and kinetic energy as functions of generalized coordinates and velocities, and derivatives of these functions constitute the equations. The approach is particularly straightforward when combined with the material description of the kinematics of deformation and motion. Considering mechanical fields as functions of coordinates in the reference configuration 35 is advantageous because an elastic body keeps "memory" of its undeformed state. Moreover, dealing with the same material volume ensures the validity of Lagrange's equations of motion. On the contrary, in fluid mechanics it becomes more efficient to observe the processes at given points in space. But the needs of modern computational mechanics go beyond the simple ideas of the past, and 40 the so-called Arbitrary Lagrangian-Eulerian (ALE) formulations are nowadays increasingly used for problems of fluid-structure interaction, material forming processes, etc. This family of methods features control volumes, which are moving in a problem-oriented manner relative to both the spatial actual state as well as the reference configuration [17. In their traditional form, ALE methods 45 imply accounting for the advection of material in the local forms of the constitutive and balance equations. An interesting application to structural mechanics, which is close to the ideas of the present study, has been presented by Hong and Ren in [18] and by Yang et al. in [19]. The authors of the mentioned papers make use of a redundant set of degrees of freedom in a finite element model with

50 additional constraints. This flexible formulation is, however, potentially more complicated and less efficient than the present one as a differential-algebraic system of equations needs to be integrated over time.

While exploiting Lagrange's equations of motion is efficient for large vibra- 
tions of structural members [20], dynamics of flexible rotors [21, etc., they cannot be directly applied to axially moving structures owing to the non-material control volume under consideration. By proper handling the flow of momentum across the boundaries of an open system, one may extend Hamilton's principle to the present case of a non-material volume [22, 23. Modelling axially moving structures, researchers frequently make use of these equations, see e.g. 24, 25.

60 Nevertheless, equations of motion for open systems obtained with the methods of Newtonian mechanics and extended Hamilton's principle may not always be identical, see comparisons and discussions in Stangl et al. 26] as well as in Chapter 5 and in Appendix E of Païdoussis [27.

Comprehensive studies on extending Lagrange's equations of motion to open systems have been reported by Irschik and Holl 28, 29, who arrived at the traditional form of equations augmented by certain integral terms over the boundaries with material flow. Taking into account the high level of abstraction of the mentioned results, in the present paper we seek complete clarity and transparency for the considered particular class of problems of axially moving continua with 70 kinematic boundary conditions. The scientifically new results of this contribution are the following.

1. We continue the research on a novel mixed Eulerian-Lagrangian kinematic description, which allows for the geometrically exact treatment of large deformations of axially moving multi-dimensional structures. Earlier [30, 31] the approach was proven to be efficient for large quasistatic deformations with no effect of inertia. An extension to dynamics is now exposed for the first time.

2. The equation of virtual work (D'Alembert's principle in dynamics), which is originally available in the material form, is transformed to the present spatial description. The new form can be applied per se for both numerical simulations as well as constructing analytical solutions, and is essentially different from the ALE methods discussed above.

3. Introducing a Galerkin-Ritz approximation of the unknown displacement field over the spatial coordinate in the non-material control domain and assuming kinematic boundary conditions, we further transform the variational equality to Lagrange's equations of motion of the second kind for the generalized degrees of freedom.

4. On an example problem with large vibrations we demonstrate the rapid mesh convergence of a simple finite element scheme and validate the solutions against available benchmark results of fully Lagrangian simulations.

In the following three sections of the paper we establish the basic notions and present the approach on the simple example of one-dimensional axial vibrations of a rod. Similar kinematic relations can be found in earlier works [14, 16, in which the analysis is focused on the differential equations of motion, and 95 not on the variational equations. The actual mechanics of transverse vibrations of axially moving strings and beams is discussed in section 5 Although the range of possible configurations is restricted by the condition that the axial coordinate must increase monotonously along the material line (see discussion 


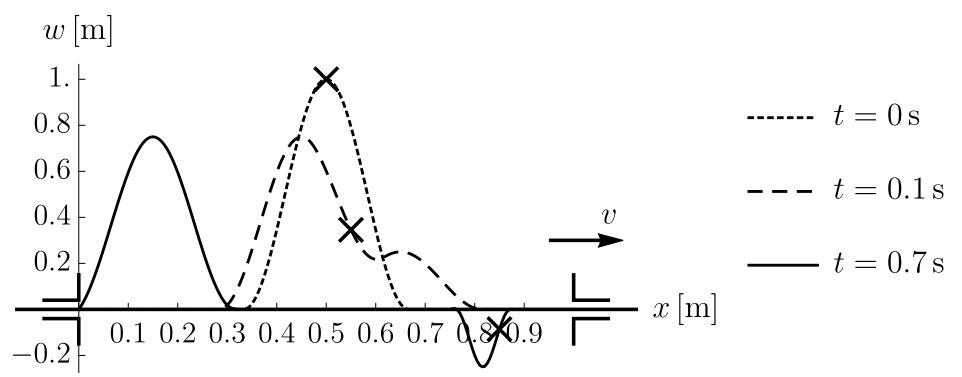

Figure 1: Wave travelling in a moving string at three different instances of time; crosses mark the same material particle

after Eq. (43)), large vibration amplitudes are treated geometrically exactly. In section 6 we present extensive numerical simulations, verifications and tests.

\section{Linear waves in a moving string}

A simple example of waves in a moving string sheds light onto the methods and effects, relevant for the subject of the paper. A particle of a string is identified by a material (Lagrangian) coordinate $s$. Assuming small transverse deflection $u$ and constant tension force, we write

$$
\begin{aligned}
& \partial_{t}^{2} u=c^{2} \partial_{s}^{2} u, \\
& \left.\partial_{s} u \equiv \frac{\partial u}{\partial s}\right|_{t=\mathrm{const}},\left.\quad \partial_{t} u \equiv \frac{\partial u}{\partial t}\right|_{s=\mathrm{const}}
\end{aligned}
$$

for a dynamic process over time $t$ with the wave speed $c$. In this known Lagrangian form we observe the mechanical field $u$ at a given material point $s$, and the wave equation features a material time derivative $\partial_{t}$. In mathematical physics, one conventionally considers Eq. (1) with conditions at the boundaries of the domain. If, however, the string is moving axially between two fixed points, then the boundary conditions need to be posed in time varying material points $s_{1,2}(t)$, which makes it essentially more difficult and challenging to solve the problem by either the established methods of mathematical physics or numerical techniques of finite differences or finite elements. Consider now a spatial coordinate $x$, along which the string is moving, see Fig. 1. Assuming a constant velocity $v$ of the gross axial motion, we find simple relations between the material and spatial coordinates and derivatives:

$$
\begin{aligned}
& x=s+v t \\
& \partial_{s} u=\partial_{x} u \equiv u^{\prime}, \quad \partial_{t} u=\dot{u}+v u^{\prime},\left.\quad \dot{u} \equiv \frac{\partial u}{\partial t}\right|_{x=\mathrm{const}} .
\end{aligned}
$$


In the following, we will mainly deal with the Eulerian form of equations, in which the unknown fields are observed at a given spatial coordinate $x$. Considering the deflection in dependence on $s$ and on $x$, we deal with two different mathematical functions. Both will be denoted with the same letter $u$ emphasizing that the mechanical fields remain the same regardless of the mathematical description. In Eq. 22 we introduce a shortened notation: a prime means a derivative with respect to $x$ and a dot denotes a time derivative at given $x$.

Now, we discuss the known transformation of the wave equation Eq. (1) to the Eulerian form. Applying the equalities Eq. (2) twice, we arrive at an equivalent boundary value problem with the new pair of variables $x$ and $t$ :

$$
\ddot{u}+2 v \dot{u}^{\prime}=\left(c^{2}-v^{2}\right) u^{\prime \prime},\left.\quad u\right|_{x=0, l}=0 .
$$

The boundary conditions are imposed in fixed points $x=0$ and $x=l$, the latter being the distance between the two points, bounding the control domain. The equation appears to be more complicated, but the boundary value problem may be solved either analytically in the form of infinite series [32], or, even easier, numerically using the method of finite differences. The example solution for the following values of parameters and the smooth initial state

$$
\begin{aligned}
& c=1 \mathrm{~m} \mathrm{~s}^{-1}, \quad l=1 \mathrm{~m}, \quad v=0.5 \mathrm{~m} \mathrm{~s}^{-1} ; \\
& t=0: \quad \dot{u}=0, \quad u= \begin{cases}u_{0}(1+\cos (6 \pi(x / l-1 / 2))) / 2, & l / 3<x<2 l / 3 \\
0, & x<l / 3 \text { or } x>2 l / 3\end{cases} \\
& u_{0}=1 \mathrm{~m}
\end{aligned}
$$

is shown for three instances of time in Fig. 1. The chosen large sample value of the amplitude $u_{0}$ lies certainly far beyond the range of applicability of the geometrically linear wave equation Eq. (1). The problem would be classical for an axially fixed string with $v=0$, see, e.g., 33: the single cosine wave with the width $l / 3$ then splits into two, running to the left and to the right with smaller but equal intensities and same width. Reflecting from the boundaries, 115 the waves would then meet again in the middle. Now, the string is moving, and the material point, which is originally in the middle of the string, is marked in Fig. 1 by a cross. We see two waves of unequal intensities, travelling with the velocities $c+v$ and $c-v$ respectively to the right and to the left and reflecting from the end points. One may alternatively consider an initial condition for the 120 material velocity $\partial_{t} u=0$ instead of $\dot{u}$ in Eq. (4), which would result into equal intensities of both waves. Last but not least, from Eq. (3) we conclude that the type of the partial differential equation changes when $v$ grows above $c$, which means loss of stiffness and dynamic instability. 


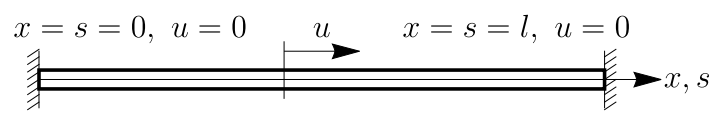

Figure 2: Axial deformation of a rod

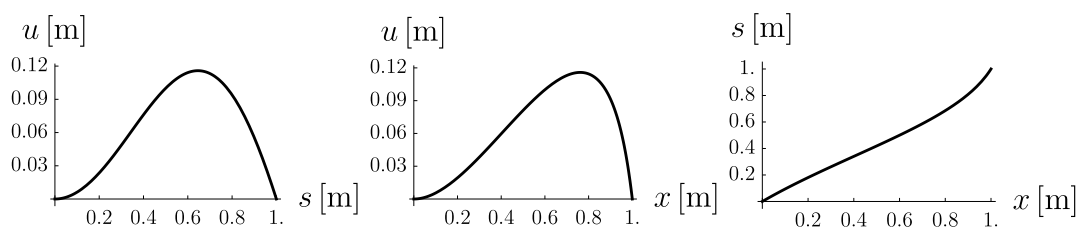

Figure 3: Sample deformation of a rod: displacement as a function of the material and spatial coordinates and the inverse mapping between the coordinates

\section{One-dimensional deformation at Lagrangian and Eulerian descrip- tions}

\subsection{Kinematics of axial deformation of a straight rod}

Now that the efficiency of Eulerian description has been demonstrated, let us address the question of its applicability for problems, in which the axial motion of particles is not known in advance. We begin with the very simple example of axial deformation of a rod, which is fixed at both ends $x=0$ and $x=l$, Fig. 2 A particle is identified by a material coordinate $s$ in the undeformed reference state. Its actual position in space is $x$, and, as mentioned earlier, the field of displacement $u=x-s$ may be considered in one of the two ways.

- In the material, or Lagrangian description we treat it as a function of the material coordinate: $u=u(s)$.

- In the spatial, or Eulerian description the displacement is a function of the spatial coordinate: $u=u(x)$.

The difference between the two descriptions vanishes in the geometrically linear formulations, when $u$ is small, but may become prominent at large deformations. Indeed, let us consider a sample deformation field

$$
u=u_{0} \frac{s}{l} \sin \frac{\pi s}{l}, \quad l=1 \mathrm{~m}, \quad u_{0}=0.2 \mathrm{~m} .
$$

This determines the mapping $x(s)=s+u$. The displacements vanish at the ends, and both the material and the spatial lengths of the rod equal $l$. The corresponding dependence of $u$ on the spatial coordinate implicitly follows from inverting the function and computing $u=x-s(x)$, which can easily be accomplished numerically, see Fig. 3 . 
In each point of the rod we have the strain $\varepsilon$, which is available for both descriptions at hand:

$$
\begin{aligned}
& x=s+u, \quad \mathrm{~d} s=\mathrm{d} x-\mathrm{d} u=\left(1-u^{\prime}\right) \mathrm{d} x, \quad u^{\prime} \equiv \partial_{x} u \\
& \varepsilon \equiv \partial_{s} u=u^{\prime} \partial_{s} x=\frac{u^{\prime}}{1-u^{\prime}} .
\end{aligned}
$$

We again introduce a short notation for the derivatives at spatial description, as the latter will dominate in the paper.

In transient processes the deformation depends on time $t$, and we compute the velocity of a material point at Eulerian description: $v=\partial_{t} x=\partial_{t}(s+u)=$ $\partial_{t} u$. By $\partial_{t}$ and (further) by the dot we correspondingly denote time derivatives at given material and spatial points as stated above in Eq. (1), Eq. (2). We compute the material time derivative of the displacement as a function of the spatial coordinate and time and arrive at the velocity after solving a simple equation:

$$
v=\partial_{t} u(x(s, t), t)=\dot{u}+u^{\prime} \partial_{t} x=\dot{u}+u^{\prime} v \quad \Rightarrow \quad v=\frac{\dot{u}}{1-u^{\prime}} ;
$$

The convective term arises also in the expression for the acceleration:

$$
w=\partial_{t} v=\dot{v}+v^{\prime} v
$$

\subsection{Strain energy and variations}

The strain energy per unit material length is

$$
U=\frac{1}{2} b \varepsilon^{2} .
$$

Setting for simplicity the tension stiffness $b=1 \mathrm{~N}$, for the sample deformation Eq. (5) we compute the total strain energy

$$
U^{\Sigma}=\int_{0}^{l} U \mathrm{~d} s=0.037899 \mathrm{Nm} .
$$

A computation in the spatial form

$$
U=\frac{1}{2} b\left(\frac{u^{\prime}}{1-u^{\prime}}\right)^{2}, \quad U^{\Sigma}=\int_{0}^{l} U\left(1-u^{\prime}\right) \mathrm{d} x=\int_{0}^{l} \frac{1}{2} \frac{u^{\prime 2}}{1-u^{\prime}} \mathrm{d} x
$$

naturally results in the same value.

Things are getting more intriguing if we consider a small variation of the displacement field and the corresponding change in the strain energy at both descriptions. To make the discussion more illustrative, let us compute how the strain energy Eq. 10) would change if the displacement field Eq. (5) obtains a particular small increment:

$$
\stackrel{\circ}{\delta}=10^{-4} u_{0} \sin \pi s / l .
$$


With the symbol $\delta$ we denote a variation of a field, which is considered at a given material coordinate $s=$ const. The corresponding increment of the energy follows as

$$
U^{\Sigma}[u+\stackrel{\circ}{\delta}]-U^{\Sigma}[u]=9.8706 \cdot 10^{-6} \mathrm{Nm} .
$$

According to variational calculus, the linearized variation of the functional $U^{\Sigma}$ follows by integrating the variation of the distributed strain energy:

$$
\stackrel{\circ}{\delta} U=b \varepsilon \stackrel{\circ}{\delta} \varepsilon=b \partial_{s} u \partial_{s} \stackrel{\circ}{\delta} u, \quad \delta U^{\Sigma}=\int_{0}^{l} \stackrel{\circ}{\delta} U \mathrm{~d} s=9.8696 \cdot 10^{-6} \mathrm{~N} \mathrm{~m} .
$$

The operations $\AA$, $\partial_{s}$ and integration with respect to $s$ are interchangeable, and the difference between the computed values in Eq. (14) and Eq. (13) lies in the higher order terms. We return to this sample computation to validate the chain of mathematical reasoning below.

In Eq. 14 we have written $\delta U^{\Sigma}$ and not $\delta^{\delta} U^{\Sigma}$ because this is an integral quantity. But as each field variable $\varphi$ may be considered as function of either $s$ or $x$, we need to differentiate between a material variation ${ }_{\delta} \varphi$ and a spatial one $\delta \varphi$, computed at $x=$ const. To establish a relation between the two variations, we consider the field as a function of the spatial coordinate and compute its material variation:

$$
\stackrel{\circ}{\delta}(x)=\stackrel{\circ}{\delta} \varphi(s+u)=\delta \varphi+\varphi^{\prime} \stackrel{\circ}{\delta} u .
$$

Applying the relation for $\varphi=u$, we find $\delta u=\left(1-u^{\prime}\right) \stackrel{\delta}{\delta}$, which resembles the expression for the velocity Eq. (7). This results in a general formula

$$
\stackrel{\circ}{\delta}=\delta \varphi+\varphi^{\prime} \frac{\delta u}{1-u^{\prime}} .
$$

Indeed, the spatial variation of the distributed strain energy Eq. (11) reads

$$
\delta U=\frac{b u^{\prime} \delta u^{\prime}}{\left(1-u^{\prime}\right)^{3}} .
$$

Simple point-wise comparison of the values at given $x$ shows, that $\delta U \neq \stackrel{\delta}{\delta}$, but evaluating $\stackrel{\circ}{\delta} U$ and $\delta U+U^{\prime} \delta u$ we obtain identical numerical values, which validates Eq. (15). Finally, an entirely Eulerian computation of the variation of the total strain energy in the form Eq. (11) becomes accessible:

$$
\delta U^{\Sigma}=\int_{0}^{l}\left(\left(1-u^{\prime}\right) \delta U-U \delta u^{\prime}\right) \mathrm{d} x
$$

the spatial variation $\delta$ is interchangeable with the integration over the spatial domain. A calculation for the considered sample fields of $u$ and its variation provides exactly the numerical value Eq. (14). 


\section{One-dimensional dynamics at Eulerian description}

155

\subsection{Problem statement and kinematic boundary conditions}

The above considerations remain valid if the rod is moving across the control domain $0 \leq x \leq l$ : material particles enter it at the left end $x=0$ and leave it at $x=l$. The displacement $u(x, t)$ in a given point in space at a certain time instant will be treated as a primary unknown in the formulation of the problem.

The material coordinates of particles at the right and left ends

$$
\left.s_{l} \equiv s\right|_{x=l},\left.\quad s_{0} \equiv s\right|_{x=0}
$$

are monotonously decreasing functions of time, and their difference $s_{l}-s_{0}$ determines the material length of the part of the rod, which is currently inside the domain. We will consider kinematic boundary conditions, when the rate of supply of material at the left end is given:

$$
-\dot{s}_{0}=\dot{u}(0, t)=\gamma=\text { const }
$$

At the right end, the mechanism of material consumption depends on the strain of the rod leaving the domain, and we may choose one of the two options.

1. The particles adhere to a moving surface immediately after leaving the control domain such, that the stretched rod is continuously transported across the boundary $x=l$ and $\left.\varepsilon\right|_{x=l+0}=\left.\varepsilon\right|_{x=l-0}$. As discussed below, $\dot{u}$ is always continuous at the boundary, which means that the velocity Eq. (7) equals the known velocity $v_{l}$ of the moving surface at the right end:

$$
\left.v\right|_{x=l}=\left.\frac{\dot{u}}{1-u^{\prime}}\right|_{x=l}=v_{l}=\text { const } .
$$

Evidently, the stationary motion is possible with

$$
\dot{u}=\gamma, \quad v=v_{l}, \quad u^{\prime}=1-\frac{\gamma}{v_{l}}
$$

everywhere inside the domain, and the difference between the rate of material supply $\gamma$ and the exit velocity $v_{l}$ defines $u^{\prime}$ and thus the strain $\varepsilon$.

2. The amount of material leaving the domain per time unit is directly known. We choose it equal to the rate of material supply to make the stationary solution possible:

$$
-\dot{s}_{l}=\dot{u}(l, t)=\gamma
$$

This sort of a boundary condition may be realized by a timing belt, moving between two rotating toothed pulleys, as it kinematically prescribes vanishing strain $\varepsilon=0$ at $x>l$ regardless of the solution in the control domain. In a stationary mode we now have $\dot{u}=\gamma$. The value of $u^{\prime}$ inside the domain is determined by the material length $s_{l}-s_{0}$, which retains its initial value. 
170 Notice that if the entire rod is considered including its parts outside the control volume, then a jump in the velocity of particles and in the strain is nearly inevitable either at the left end in the case of the first type boundary condition or at both ends for the second type. This means that the mentioned toothed pulleys will contact the belt by forces, which are concentrated at just outermost 175 single teeth; the point is discussed in more detail in [14, 16. The discontinuities, however, play little role for the subsequent analysis, as at a time instant just the active material domain $s_{0} \leq s \leq s_{l}$ is considered, in which no discontinuities are expected as long as all the influences on the system are smooth. Anyway, the mapping $x(s)$ remains continuous for the entire rod, which means continuity of $u$ with respect to both $s$ and $x$. In its own turn, it means that $\dot{u}$ may not undergo jumps at a particular point in space over any finite period of time, including the boundary points.

\subsection{Transformation of the equation of virtual work to the Eulerian form}

Variational equation of balance of virtual work, which is called D'Alembert's principle in dynamics, is often considered being a basic law of mechanics 34 , 35, 36. No external forces act on the rod, and for a segment within the control volume the equation reads

$$
\int_{s_{0}}^{s_{l}}(\rho w \stackrel{\circ}{\delta} u+\stackrel{\circ}{\delta} U) \mathrm{d} s=0 .
$$

Here $\rho$ is the mass density per unit material length. The boundary terms do not enter the equation because of the kinematic nature of the boundary conditions Eq. (20), Eq. 21), Eq. (23): the variation $\delta u$ is considered at "frozen" kinematic conditions and thus shall vanish at $s=s_{0, l}$. Indeed, the second type of boundary conditions implies that $u$ is a given function of time at the end points and cannot be varied, while the first type results in an own differential equation for $u_{l}(t)$, which needs to be integrated over time simultaneously with the equations of the dynamics of the rest of the system. In both cases the forces acting at these points produce no virtual work. For free boundaries with force boundary conditions we refer to Irschik and Holl [28, 29.

We aim at transforming Eq. (24) to a variational equation for the spatial form of the field $u$. As discussed above, the integral of $\delta U$ results into the variation of the total strain energy, computed at spatial description from Eq. (11). It remains to transform the first dynamic term:

$$
\int_{s_{0}}^{s_{l}} \rho w \stackrel{\circ}{\delta} u \mathrm{~d} s=\int_{0}^{l} \rho w \frac{1-u^{\prime}}{1-u^{\prime}} \delta u \mathrm{~d} x=\int_{0}^{l} \rho w \delta u \mathrm{~d} x .
$$

The factors resulting from the change of integration variable and transformation of the variation to the spatial form mutually cancel one another, and the integral over the spatial domain retains the familiar look (which is not the case in the two-dimensional analysis, see section 5.3p. This immediately leads to the sought non-material variational equation

$$
\int_{0}^{l} \rho w \delta u \mathrm{~d} x+\delta U^{\Sigma}=0
$$


Along with the expressions for the acceleration and for the strain energy Eq. (8), Eq. (11), we now have a variational problem for the unknown field $u(x, t)$. Moreover, using the expressions Eq. (17), Eq. (18) for the spatial variation of the strain energy and integrating by parts to transform $\delta u^{\prime}$ to $\delta u$, we arrive at the known differential equation of motion in the spatial form

$$
\rho w=\frac{Q^{\prime}}{1-u^{\prime}}, \quad Q=b \varepsilon .
$$

It deserves to be noted that the spatial form of $w$ includes the derivative $v^{\prime}$, and the velocity itself features $u^{\prime}$ in Eq. (7), which means that the variational equation shall be considered for smooth fields of $u$.

\subsection{Ritz-Galerkin approximation}

We seek the unknown field of displacement as a linear combination of given shape functions $u_{k}(x)$ with time dependent coefficients $q_{k}(t)$ :

$$
u=\sum_{k=0}^{n} u_{k}(x) q_{k}(t), \quad u^{\prime}=\sum_{k=0}^{n} u_{k}^{\prime} q_{k}, \quad \dot{u}=\sum_{k=0}^{n} u_{k} \dot{q}_{k}, \quad \delta u=\sum_{k=0}^{n} u_{k} \delta q_{k} .
$$

The system $u_{k}(x)$ shall be linear independent and complete, such that the exact solution can be approached by increasing the number of terms $n$. Substituting in Eq. (26) and collecting the coefficients at independent variations $\delta q_{k}$, we obtain a system of ordinary differential equations for the generalized degrees of freedom $q_{k}(t)$, which is typical for Galerkin's procedure with the same sets of trial and test functions. Later we shall use the same approximation in the energy functional, which is closer to the idea of the Ritz method of minimization.

The second type of boundary conditions Eq. (21), Eq. (23) implies that the functions $u(0, t)$ and $u(l, t)$ are kinematically prescribed. We demand that all shape functions vanish at the end points besides the very first and the very last ones:

$$
u_{k}(0)=\left\{\begin{array}{ll}
1, & k=0 \\
0, & k>0
\end{array} \quad, \quad u_{k}(l)=\left\{\begin{array}{ll}
0, & k<n \\
1, & k=n
\end{array} .\right.\right.
$$

These properties are fulfilled by a finite element approximation with shape functions, which differ from 0 just in a part of the domain, namely in one or two adjacent finite elements. Positioning the first and the last nodes at the left and right ends of the domain results in $q_{0}(t)=u(0, t)$ and $q_{n}(t)=u(l, t)$, such that we can exclude the first and the last generalized degrees of freedom from the entire set and replace them by given functions of time. The rest of degrees of freedom with indices $k=1 \ldots n-1$ (active set) acquires differential equations from the above Galerkin-type procedure. This corresponds to the discussion of the kinematic nature of the boundary conditions and vanishing variation of displacement in the end points shortly after Eq. 24.

The situation is slightly different with the first type of boundary condition at the right end. Now, Eq. 21) provides an additional first order differential 
equation for $q_{n}$, which needs to be integrated over time simultaneously with the other $k-1$ second order equations of dynamics.

It remains to notice that the requirement of smoothness of trial functions for the variational equation Eq. (26), expressed in the end of section 4.2 implies that the shape functions $u_{k}(x)$ are to be continuous including the first derivative. Although this requirement is relaxed in the transformed formulation below, which does not deal with accelerations, we nevertheless use $C^{1}$ continuous finite element approximations in the numerical simulations for the sake of 225 rapid convergence.

\subsection{Lagrange's equations of motion of the second kind for non-material domain}

While the variational equation Eq. 24 can successfully be used for finite element simulations, an implementation may be tedious: the dynamical terms in the differential equations result from the integration of products of $\rho w$ with ${ }_{230}$ the shape functions, which requires a non-trivial assembly procedure. On the contrary, the form of Lagrange's equations of motion of the second kind, which are determined by just two energy functions, is attractive because of its simplicity and easiness of implementation using computer algebra software. Despite the open system with mass exchange at hand, we expect the traditional form of Lagrange's equations of motion to still hold based upon the research work by Irschik and Holl 28, 29. They treated dynamics of a deformable solid with a non-material control volume and with mass flow over the boundary. As a result it was shown that the equations are to be augmented by boundary integral terms, in which the distributed kinetic energy is differentiated with respect to 240 generalized velocities $\dot{q}_{k}$. These terms vanish in the problem at hand, as the material velocities of the two boundary points $\left.v\right|_{x=0, l}$ depend generally on the time derivatives $\dot{u}(0, t)=\dot{q}_{0}$ and $\dot{u}(l, t)=\dot{q}_{n}$, which do not belong to the active set of degrees of freedom and are governed by own differential equations, and on the strain in these points, which is independent from the generalized velocities

$245 \dot{q}_{k}$. Nevertheless, the abstract level of argumentation of [28, 29] and non-evident correspondence to the present case of a structural member with mixed EulerianLagrangian description (when the transverse motion is considered below) make it reasonable to explicitly demonstrate the equivalence between the variational equation of virtual work Eq. (24) and the Lagrange's equations of motion.

We proceed by transforming the expression of the functional of the total kinetic energy $T^{\Sigma}$ to the spatial form:

$$
T^{\Sigma}[u, \dot{u}]=\int_{s_{0}}^{s_{l}} \frac{1}{2} \rho v^{2} \mathrm{~d} s=\int_{0}^{l} \frac{1}{2} \rho v^{2}\left(1-u^{\prime}\right) \mathrm{d} x=\int_{0}^{l} \frac{1}{2} \frac{\rho \dot{u}^{2}}{1-u^{\prime}} \mathrm{d} x,
$$

the expression for $v$ in Eq. (7) has been used. The last explicit integral determines the function $T^{\Sigma}\left(q_{k}, \dot{q}_{k}\right)$ for a particular Ritz-Galerkin approximation 
Eq. 28). Now we compute

$$
\begin{aligned}
& \frac{\partial v}{\partial \dot{q}_{k}}=\frac{1}{1-u^{\prime}} \frac{\partial \dot{u}}{\partial \dot{q}_{k}}=\frac{u_{k}}{1-u^{\prime}} \\
& \frac{\partial T^{\Sigma}}{\partial \dot{q}_{k}}=\int_{0}^{l} \rho v \frac{\partial v}{\partial \dot{q}_{k}}\left(1-u^{\prime}\right) \mathrm{d} x=\int_{0}^{l} \rho v u_{k} \mathrm{~d} x \\
& \frac{\mathrm{d}}{\mathrm{d} t}\left(\frac{\partial T^{\Sigma}}{\partial \dot{q}_{k}}\right)=\int_{0}^{l} \rho \dot{v} u_{k} \mathrm{~d} x .
\end{aligned}
$$

Computing the second term in Lagrange's equations, we integrate by parts and take into account that $u_{k}(0)=u_{k}(l)=0$ for all active degrees of freedom $q_{k}$, $k=1 \ldots n-1$ :

$$
-\frac{\partial T^{\Sigma}}{\partial q_{k}}=-\int_{0}^{l} \frac{1}{2} \frac{\rho \dot{u}^{2}}{\left(1-u^{\prime}\right)^{2}} \frac{\partial u^{\prime}}{\partial q_{k}} \mathrm{~d} x=-\int_{0}^{l} \frac{1}{2} \rho v^{2} u_{k}^{\prime} \mathrm{d} x=\int_{0}^{l} \rho v v^{\prime} u_{k} \mathrm{~d} x .
$$

Comparing now to the expression for the acceleration Eq. (8) and using

$$
\delta U^{\Sigma}=\frac{\partial U^{\Sigma}}{\partial q_{k}} \delta q_{k}
$$

we finally bring Eq. (26) to the desired form:

$$
\int_{0}^{l} \rho w \delta u \mathrm{~d} x+\delta U^{\Sigma}=\sum_{k=1}^{n-1}\left(\frac{\mathrm{d}}{\mathrm{d} t}\left(\frac{\partial T^{\Sigma}}{\partial \dot{q}_{k}}\right)-\frac{\partial T^{\Sigma}}{\partial q_{k}}+\frac{\partial U^{\Sigma}}{\partial q_{k}}\right) \delta q_{k}=0 .
$$

The coefficients at independent variations $\delta q_{k}$ must vanish, which provides a system of differential equations for the generalized coordinates in the form of Lagrange's equations of motion of the second kind.

We validated the above arguments in a series of numerical experiments, using both the discussed non-material finite element scheme and a simple Lagrangian model with many lumped mass particles and springs. The motion of particles was kinematically prescribed before the control domain at $x \leq 0$ and after it, where $x \geq l$. An artificial small viscosity parameter prevented the model from growing numerically induced oscillations, which are excited by periodic switching of the particles from the kinematically driven state to the "free flight" mode and back. For both types of boundary conditions, at small strains and at large strains within the control volume the results of simulations of both models converged to the same solutions. With just a few $C^{1}$ continuous finite elements (see section 5.4) we achieved the level of accuracy, which required hundreds of particles within the domain when using the model with lumped masses.

\section{In-plane dynamics of axially moving strings and beams}

\subsection{Formulation of a problem}

We extend the above simple example to the two-dimensional case of planar motion of a string or a beam across a given domain, see Fig. 4. The material 

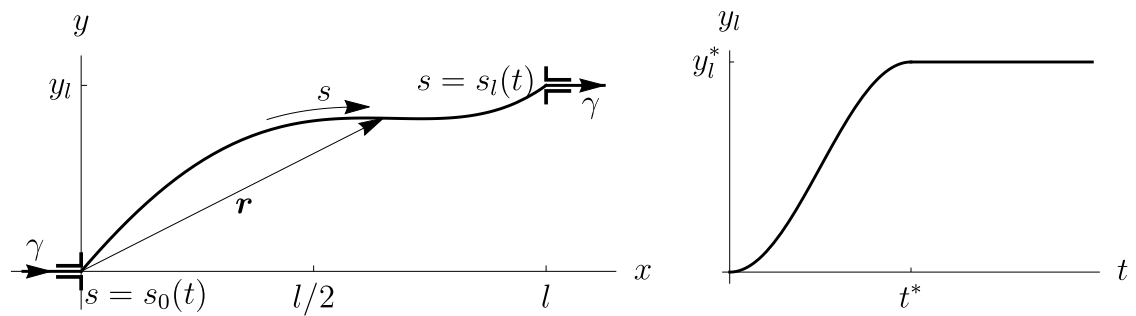

Figure 4: In-plane dynamics of an axially moving string (left), kinematically prescribed transverse motion of the right end (right)

enters the domain at the left end $x=0$ and leaves it at the right one $x=l$ with the same rate $\gamma$, in analogy to the one-dimensional counterpart Eq. (20), Eq. 23p. The kinematically prescribed time-varying transverse deflection of the end point

$$
y_{l}(t)= \begin{cases}\frac{1}{2} y_{l}^{*}\left(1-\cos \frac{\pi t}{t^{*}}\right), & t \leq t^{*} \\ y_{l}^{*}, & t>t^{*}\end{cases}
$$

brings the system into a complicated in-plane motion with coupled axial and transverse dynamics when $\dot{y}_{l}$ is not small.

Although the equations of both string and beam dynamics at finite deformations are well established at Lagrangian description [14, 20, 34, 37, 38, it is a numerically challenging task to solve them with the boundary conditions moving across the material domain. Thus, an attempt to impose kinematic constraints at moving points of a finite element mesh leads inevitably to numerically induced oscillations in the solution. Therefore, in the following we will again use D'Alembert's form of the principle of virtual work as a starting point. The necessary expression of the strain energy per unit material length $s$ reads

$$
U=\frac{1}{2} b \varepsilon^{2}+\frac{1}{2} a \kappa^{2},
$$

in which $b$ is the tension stiffness, $\varepsilon$ is the strain, $a$ is the bending stiffness and $\kappa$ is the material curvature of an initially straight non-shearable, but extensible beam.

At material description, we consider the displacement from an initially straight horizontal reference configuration

$$
\mathbf{u}=u_{x} \mathbf{i}+u_{y} \mathbf{j}
$$

being a function of the material coordinate $s$ and time $t$, such that the actual position vector of a particle is

$$
\mathbf{r}=x \mathbf{i}+y \mathbf{j}=s \mathbf{i}+\mathbf{u}, \quad x=s+u_{x}, \quad y=u_{y} ;
$$

the unit vectors in the directions of the coordinate axes are respectively called $\mathbf{i}$ and $\mathbf{j}$. The longitudinal strain measure reads

$$
\varepsilon=\left|\partial_{s} \mathbf{r}\right|-1=\sqrt{\left(\partial_{s}\left(s+u_{x}\right)\right)^{2}+\left(\partial_{s} u_{y}\right)^{2}}-1 .
$$


The bending strain measure differs from the geometrical curvature when extension is taken into account because the geometrical and the material lengths are not identical. From the relations for the unit tangent and normal vectors $\mathbf{t}$ and $\mathbf{n}$,

$$
\partial_{s} \mathbf{r}=(1+\varepsilon) \mathbf{t}, \quad \partial_{s} \mathbf{t}=-\kappa \mathbf{n}, \quad \mathbf{t} \cdot \mathbf{t}=1, \quad \mathbf{n} \cdot \mathbf{n}=1, \quad \mathbf{t} \cdot \mathbf{n}=0
$$

we find

$$
\kappa=\frac{\left(1+\partial_{s} u_{x}\right) \partial_{s}^{2} u_{y}-\partial_{s} u_{y} \partial_{s}^{2} u_{x}}{\left(1+\partial_{s} u_{x}\right)^{2}+\partial_{s} u_{y}^{2}}
$$

When considering a beam, one needs to take into account not only the bending energy, but also two additional boundary conditions. Assuming the parts of the beam outside of the control domain to be parallel to the axial direction, we demand that the tangent vector remains continuous:

$$
\left.\mathbf{t}\right|_{s_{0, l}}=\left.\mathbf{i} \Rightarrow \partial_{s} u_{y}\right|_{s_{0, l}}=0 .
$$

The configuration in Fig. 4 corresponds to the case of a string, which may violate these conditions.

\subsection{Kinematics at mixed Eulerian-Lagrangian description}

The simple idea of the mixed Eulerian-Lagrangian description is a change of variable. Instead of the unknown fields $u_{x}(s, t), u_{y}(s, t)$, we seek the material coordinate and the transverse displacement as functions of the axial spatial coordinate and time:

$$
s=s(x, t)=x-u_{x}(x, t), \quad u_{y}=u_{y}(x, t)
$$$$
\text { (1) }
$$
pricated types of motion like e.g. a looped trajectory of a belt drive requires a problem-oriented curvilinear coordinate system, which again allows decoupling the spatial coordinates into the Eulerian (circumferential) and the Lagrangian one. Alternatively, the general formulation with a redundant set of unknowns [18, 19] may be applied, which results in a system of differential-algebraic equations with complicated constraint conditions.

Despite similarity to the previously considered one-dimensional case, the presence of the second dimension and the vectorial nature of the displacement, velocity and acceleration make the mathematics more tedious now. Again introducing short notation for the derivatives with the pair of variables $x, t$ similar to Eq. 22 and using

$$
\mathrm{d} s=\mathrm{d} x-\mathrm{d} u_{x}=\left(1-u_{x}^{\prime}\right) \mathrm{d} x, \quad \partial_{s}(\ldots)=\left(1-u_{x}^{\prime}\right)^{-1}(\ldots)^{\prime},
$$


we rewrite the strain measures Eq. (39), Eq. (41) to the spatial form

$$
\varepsilon=\frac{\sqrt{1+u_{y}^{\prime 2}}}{1-u_{x}^{\prime}}-1, \quad \kappa=\frac{u_{y}^{\prime \prime}}{\left(1+u_{y}^{\prime 2}\right)\left(1-u_{x}^{\prime}\right)} .
$$

Now we address the velocity of a particle and compute it in the mixed description similar to the purely Eulerian one-dimensional case Eq. (7):

$$
\begin{aligned}
& \partial_{t} x=\partial_{t} u_{x}(x, t)=\dot{u}_{x}+u_{x}^{\prime} \partial_{t} x \Rightarrow \partial_{t} x=\frac{\dot{u}_{x}}{1-u_{x}^{\prime}}, \\
& \mathbf{v}=\partial_{t} \mathbf{u}(x, t)=\dot{\mathbf{u}}+\mathbf{u}^{\prime} \partial_{t} x=\dot{\mathbf{u}}+\mathbf{u}^{\prime} \frac{\dot{u}_{x}}{1-u_{x}^{\prime}}
\end{aligned}
$$

The acceleration results into

$$
\mathbf{w}=\dot{\mathbf{v}}+\mathbf{v}^{\prime} \frac{\dot{u}_{x}}{1-u_{x}^{\prime}}
$$

and with similar mathematics we relate the material variation $\delta$ at fixed material point and the spatial one $\delta$ at given $x$ :

$$
\stackrel{\circ}{\delta} \varphi=\delta \varphi+\varphi^{\prime} \frac{\delta u_{x}}{1-u_{x}^{\prime}} .
$$

\subsection{Mixed Eulerian-Lagrangian form of the variational equation of virtual work}

We begin with the material form of the variational equation of virtual work for the segment of the string (beam) $s_{0} \leq s \leq s_{l}$, which is currently in the active domain:

$$
\int_{s_{0}}^{s_{l}}(\rho \mathbf{w} \cdot \stackrel{\circ}{\delta} \mathbf{u}+\stackrel{\circ}{\delta} U) \mathrm{d} s=0 .
$$

Similar to Eq. (24), the variation $\delta \mathbf{u}$ vanishes at the current boundaries owing to the kinematic boundary conditions, and there is no virtual work produced by the interaction forces with the parts of the continuum outside the active domain. In the case of a beam, the rotation of the particles $s_{0, l}$ is prescribed kinematically by Eq. 42 , and the moment interaction also results in no virtual work.

Transforming the elastic term in Eq. 49. to the spatial form is simple:

$$
\int_{s_{0}}^{s_{l}} \stackrel{\circ}{\delta} U \mathrm{~d} s=\delta U^{\Sigma}, \quad U^{\Sigma}=\int_{s_{0}}^{s_{l}} U \mathrm{~d} s=\int_{0}^{l} U\left(1-u_{x}^{\prime}\right) \mathrm{d} x .
$$

The distributed strain energy $U$ remains a quadratic form Eq. (36) of the strain measures Eq. 45). The inertial term in Eq. 49) reads

$$
\int_{s_{0}}^{s_{l}} \rho \mathbf{w} \cdot \stackrel{\circ}{\delta} \mathbf{u} \mathrm{d} s=\int_{0}^{l} \rho \mathbf{w} \cdot\left(\left(1-u_{x}^{\prime}\right) \delta \mathbf{u}+\mathbf{u}^{\prime} \delta u_{x}\right) \mathrm{d} x
$$


besides Eq. (44) we also used the transformation rule for the variations Eq. (48). Substituting Eq. (50) and Eq. (51) in Eq. (49), we obtain a non-material variational equation. Similar to the one-dimensional counterpart Eq. (26), it can be used in a numerical procedure with a suitable Ritz-Galerkin approximation of the unknown displacement $\mathbf{u}(x, t)$. The procedure is, however, more elegant, its implementation is easier and the resulting system of ordinary differential equations possesses better numerical properties with the form of Lagrangian equations of motion of the second kind.

\subsection{Finite element approximation}

Before we proceed to further mathematical transformations, let us discuss the applied finite element approximation of the unknown field of displacements:

$$
\mathbf{u}=\sum_{k=0}^{n}\left(u_{k}(x) q_{k, 1}(t) \mathbf{i}+u_{k}(x) q_{k, 2}(t) \mathbf{j}\right)=\sum_{k=0}^{n} \sum_{\alpha=1}^{2} \mathbf{u}_{k, \alpha}(x) q_{k, \alpha}(t) .
$$

We approximate both components of the displacement field independently: the degrees of freedom $q_{k, 1}$ affects the axial component $u_{x}$, and $q_{k, 2}$ are responsible for the transverse deflection $u_{y}$. The same piecewise-cubic shape functions $u_{k}(x)$ are used for both components, which allows achieving $C^{1}$ inter-element continuity. The latter is important for the beam model with the requirement of a smooth approximation of the geometry, and improves the rate of mesh convergence. Considering a regular mesh with equal finite element sizes and a local coordinate $\xi$ on each finite element, which varies from -1 to 1 , we have a linear mapping from $\xi$ to $x$ and use four cubic shape functions $u_{i}^{\mathrm{el}}(\xi)$ with the properties

$$
\begin{aligned}
& \left.u_{i}^{\mathrm{el}}\right|_{\xi=-1}=\delta_{1, i},\left.\partial_{\xi} u_{i}^{\mathrm{el}}\right|_{\xi=-1}=\delta_{2, i},\left.u_{i}^{\mathrm{el}}\right|_{\xi=1}=\delta_{3, i},\left.\partial_{\xi} u_{i}^{\mathrm{el}}\right|_{\xi=1}=\delta_{4, i} ; \\
& \delta_{j, i}=\left\{\begin{array}{ll}
1, & i=j \\
0, & i \neq j
\end{array} ;\right.
\end{aligned}
$$

the four conditions uniquely determine four coefficients of cubic polynomials, which often find use in the models of classical rods and shells [20, 37, 39]. The global shape functions $u_{k}(x)$ are constructed from the local ones $u_{i}^{\mathrm{el}}(\xi)$, degrees of freedom $q_{k, \alpha}$ determine either the value or the derivative of the corresponding component of $\mathbf{u}$ in a node. Each node has now four degrees of freedom, and $n$ is twice the number of nodes in the finite element model. The boundary conditions in the first and the last nodes,

$$
\left.\dot{u}_{x}\right|_{x=0, l}=\gamma,\left.\quad u_{y}\right|_{x=0}=0,\left.\quad u_{y}\right|_{x=l}=y_{l}(t)
$$

kinematically prescribe some of the functions $q_{k, \alpha}$, which thus fall out from the set of active degrees of freedom in the model. In the case of a beam, additional two boundary conditions

$$
\left.\partial_{x} u_{y}\right|_{x=0, l}=0,
$$

follow from Eq. 42 and further reduce the number of active degrees of freedom. 


\subsection{Lagrange's equation of motion of the second kind}

Consider a particular degree of freedom $q_{k, \alpha}$ and its shape function. As the mathematics below is independent from the indices $k$ and $\alpha$, we shorten the equations within this section by denoting $q \equiv q_{k, \alpha}$ and $\varphi(x)=\varphi_{x} \mathbf{i}+\varphi_{y} \mathbf{j} \equiv \mathbf{u}_{k, \alpha}$. The shape function vanishes at both ends for all active degrees of freedom:

$$
\varphi(0)=\varphi(l)=0 .
$$

For the sake of conciseness we treat other degrees of freedom as fixed and write

$$
\delta \mathbf{u}=\varphi \delta q, \quad \dot{\mathbf{u}}=\varphi \dot{q}, \quad \frac{\partial \mathbf{u}}{\partial q}=\frac{\partial \dot{\mathbf{u}}}{\partial \dot{q}}=\varphi .
$$

The right-hand side of Eq. (51) equals $A \delta q$, in which

$$
\begin{aligned}
A= & \int_{0}^{l} \rho \mathbf{w} \cdot\left(\left(1-u_{x}^{\prime}\right) \boldsymbol{\varphi}+\mathbf{u}^{\prime} \varphi_{x}\right) \mathrm{d} x= \\
& =\int_{0}^{l} \rho\left(\dot{\mathbf{v}} \cdot \boldsymbol{\varphi}\left(1-u_{x}^{\prime}\right)+\mathbf{v}^{\prime} \cdot \varphi \dot{u}_{x}+\dot{\mathbf{v}} \cdot \mathbf{u}^{\prime} \varphi_{x}+\mathbf{v}^{\prime} \cdot(\mathbf{v}-\dot{\mathbf{u}}) \varphi_{x}\right) \mathrm{d} x
\end{aligned}
$$

we have used expressions for the velocity Eq. (46) and for the acceleration Eq. (47). Similar to the one-dimensional case Eq. (30)-Eq. (34), we aim now at showing the equivalence of the dynamic part of the equation of motion $A$ to the left-hand side of the Lagrange's equation of motion of the second kind

$$
\frac{\mathrm{d}}{\mathrm{d} t}\left(\frac{\partial T^{\Sigma}}{\partial \dot{q}}\right)-\frac{\partial T^{\Sigma}}{\partial q}=-\frac{\partial U^{\Sigma}}{\partial q}
$$

with the kinetic energy of the control domain

$$
T^{\Sigma}=\int_{0}^{l} \frac{1}{2} \rho \mathbf{v} \cdot \mathbf{v}\left(1-u_{x}^{\prime}\right) \mathrm{d} x .
$$

We begin by computing

$$
\begin{aligned}
& \frac{\partial T^{\Sigma}}{\partial \dot{q}}=\int_{0}^{l} \rho \mathbf{v} \cdot \frac{\partial \mathbf{v}}{\partial \dot{q}}\left(1-u_{x}^{\prime}\right) \mathrm{d} x=\int_{0}^{l} \rho \mathbf{v} \cdot\left(\varphi\left(1-u_{x}^{\prime}\right)+\mathbf{u}^{\prime} \varphi_{x}\right) \mathrm{d} x \\
& B=\frac{\mathrm{d}}{\mathrm{d} t}\left(\frac{\partial T^{\Sigma}}{\partial \dot{q}}\right)=\int_{0}^{l} \rho\left(\dot{\mathbf{v}} \cdot \boldsymbol{\varphi}\left(1-u_{x}^{\prime}\right)-\mathbf{v} \cdot \varphi \dot{u}_{x}^{\prime}+\mathbf{v} \cdot \dot{\mathbf{u}}^{\prime} \varphi_{x}+\dot{\mathbf{v}} \cdot \mathbf{u}^{\prime} \varphi_{x}\right) \mathrm{d} x= \\
& =\int_{0}^{l} \rho\left(\dot{\mathbf{v}} \cdot \varphi\left(1-u_{x}^{\prime}\right)+\mathbf{v}^{\prime} \cdot \varphi \dot{u}_{x}+\dot{\mathbf{v}} \cdot \mathbf{u}^{\prime} \varphi_{x}+\mathbf{v} \cdot \varphi^{\prime} \dot{u}_{x}+\mathbf{v} \cdot \dot{\mathbf{u}}^{\prime} \varphi_{x}\right) \mathrm{d} x
\end{aligned}
$$

we have integrated by parts, and the boundary terms vanish because of Eq. (56). Several terms in $A$ and $B$ are already identical. We proceed with

$$
\begin{aligned}
C= & \frac{\partial T^{\Sigma}}{\partial q}=\int_{0}^{l} \rho\left(-\frac{1}{2} \mathbf{v} \cdot \mathbf{v} \frac{\partial u_{x}^{\prime}}{\partial q}+\mathbf{v} \cdot \frac{\partial \mathbf{v}}{\partial q}\left(1-u_{x}^{\prime}\right)\right) \mathrm{d} x= \\
& =\int_{0}^{l} \rho\left(-\frac{1}{2} \mathbf{v} \cdot \mathbf{v} \varphi_{x}^{\prime}+\mathbf{v} \cdot\left(\varphi^{\prime} \dot{u}_{x}+\frac{\mathbf{u}^{\prime} \dot{u}_{x} \varphi_{x}^{\prime}}{1-u_{x}^{\prime}}\right)\right) \mathrm{d} x .
\end{aligned}
$$


Now we keep on integrating by parts and recall Eq. 446):

$$
C=\int_{0}^{l} \rho\left(\varphi_{x} \mathbf{v} \cdot \mathbf{v}^{\prime}+\mathbf{v} \cdot\left(\varphi^{\prime} \dot{u}_{x}+(\mathbf{v}-\dot{\mathbf{u}}) \varphi_{x}^{\prime}\right)\right) \mathrm{d} x .
$$

Finally, we build the difference and collect the terms:

$$
\begin{gathered}
A-(B-C)=\int_{0}^{l} \rho\left(\left(\mathbf{v}^{\prime} \varphi_{x}+\mathbf{v} \varphi_{x}^{\prime}\right) \cdot(\mathbf{v}-\dot{\mathbf{u}})+\varphi_{x} \mathbf{v} \cdot \mathbf{v}^{\prime}-\varphi_{x} \mathbf{v} \cdot \dot{\mathbf{u}}^{\prime}\right) \mathrm{d} x= \\
=\int_{0}^{l} \rho\left(\varphi_{x} \mathbf{v} \cdot(\mathbf{v}-\dot{\mathbf{u}})\right)^{\prime} \mathrm{d} x=0,
\end{gathered}
$$

again because of Eq. (56). This proves the equivalence of the system of ordinary differential equations Eq. (59) to the variational formulation of D'Alembert's principle of virtual work for the considered finite element approximation with kinematic boundary conditions.

\section{Numerical simulations and verification}

\subsection{Particular setup and implementation}

The finite element scheme including the time integration was implemented in the Wolfram Mathematico ${ }^{1}$ environment. With $n_{\mathrm{el}}$ being the number of elements in the model, we have $n_{\mathrm{el}}+1$ nodes with $4\left(n_{\mathrm{el}}+1\right)$ degrees of freedom $q_{k, \alpha}$. Depending on the type of the structure (string or beam), either 4 or 6 degrees of freedom are prescribed by the boundary conditions and excluded from the active

315 set. Using the benefits of the computer algebra software, we derive expressions for the strain measures Eq. (45) and for the velocity Eq. (46) in a given point $\xi$ of an element as functions of the degrees of freedom of the adjacent nodes and their time derivatives. The total strain energy Eq. (50) and kinetic energy Eq. (60) were assembled using a Gaussian quadrature rule with 3 integration points per element, which is sufficient to avoid spurious modes in the solution. Substituting further the known time variations for the kinematically prescribed degrees of freedom, we construct the system of second order differential equations for the transient dynamics in the form Eq. (59). With the values of the numerical parameters, initial values and time derivatives of degrees of freedom 325 we integrate the equations over time using standard NDSolve routine and obtain results, which are ready for post-processing. Because of the complexity of the system, Mathematica experiences problems when trying to resolve the higher-order derivatives of the unknowns. This is fixed by explicitly instructing NDSolve treating the problem as a system of differential-algebraic equations using the setting Method $\rightarrow$ \{"EquationSimplification" $\rightarrow$ "Residual" $\}$. Using a DAE solver is anyway necessary for problems with the first kind of boundary condition at the right end.

\footnotetext{
${ }^{1}$ http://www.wolfram.com
} 
As a first simple test, we considered the linear problem of wave propagation in a moving string, discussed in the motivation example, section 2 Setting the numerical values and the initial conditions in accordance with Eq. (4) (such that the tension in the string, its density and stiffness result in $c=1 \mathrm{~m} \mathrm{~s}^{-1}$, and using small amplitude of the transverse deflection $w_{0}$ to remain in the linear range), we were able to reproduce the results of Fig. 1 with a high level of accuracy even using small $n_{\mathrm{el}}$.

The presented below numerical experiments feature the following parameters of the model with large deformations:

$$
\begin{aligned}
& l=1 \mathrm{~m}, \quad b=1 \mathrm{~N}, \quad a=0.05^{2} / 12 \mathrm{~N} \mathrm{~m}^{2}, \quad \rho=1 \mathrm{~kg} \mathrm{~m}^{-1}, \\
& \gamma=0.1 \mathrm{~m} \mathrm{~s}^{-1}, \quad \varepsilon_{0}=0.1, \quad t^{*}=5 \mathrm{~s}, \quad y_{l}^{*}=0.4 \mathrm{~m} .
\end{aligned}
$$

Scalability of the model allows testing the numerical scheme for unit tension stiffness and inertia, and the bending stiffness (if we deal with a beam model) corresponds to a square cross-section of a beam with the height 20 times less than the length of the control domain. The structure is pre-stressed, and the initial tension strain $\varepsilon_{0}$ determines the initial material length of the active part of the string or beam $s_{l}-\left.s_{0}\right|_{t=0}$, which remains constant during the simulation as long as we consider the second kind of the boundary conditions Eq. (23). In the beginning of the simulation, the structure is moving steadily with

$$
t=0: \quad \dot{\mathbf{u}}=\gamma \mathbf{i}, \quad \mathbf{u}^{\prime}=\frac{\varepsilon_{0}}{1+\varepsilon_{0}} \mathbf{i} .
$$

In the case of a string, the initial velocity of transverse waves is determined by the pre-tension and for the given numerical parameters can be estimated as $\sqrt{0.1} \mathrm{~m} \mathrm{~s}^{-1}$, which is comparable to the rate of material supply $\gamma$. The transverse motion of the right end $y_{l}(t)$ according to Eq. (35) increases the general tension level and the wave velocity over the time period $0 \leq t \leq t^{*}$.

\subsection{Example solutions}

Taking $n_{\mathrm{el}}=10$, we sequentially solved four transport problems with the following settings.

- The moving string with material consumption rate $\gamma$ at the right end (second kind of the boundary condition Eq. (23) ) is considered as a basic reference example.

- We reduced the axial transport rate $\gamma$ by half to demonstrate its effect on the solution.

- We switched to the first kind of the boundary condition at the right end Eq. (21), thus prescribing the horizontal component of the material velocity of particles $v_{l}$. From the velocity Eq. (7) and the strain Eq. (6) we find the initial velocity of the string, which we hold constant at the right end during the entire simulation:

$$
v_{l}=\gamma\left(1+\varepsilon_{0}\right) .
$$




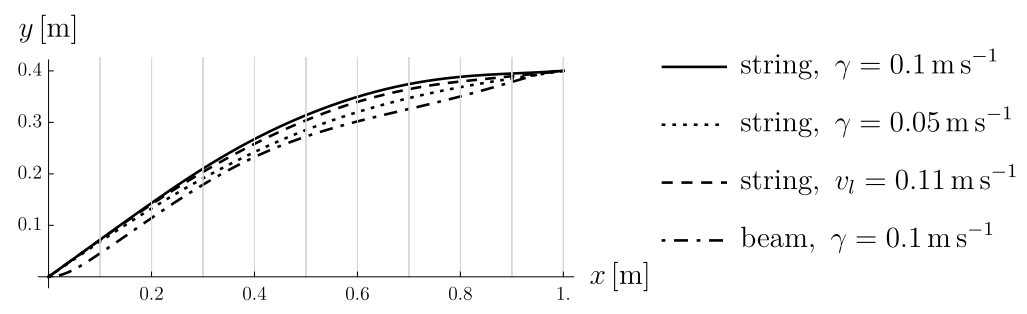

Figure 5: Deformed structure in the control volume at $t=10$ for the cases of a moving string, a string with twice slower axial transport, a string with the first type of boundary condition at the right end (horizontal material velocity $v_{l}$ prescribed instead of the rate of material consumption $\gamma$ ) and a beam

- Introducing the bending stiffness and using the additional boundary conditions Eq. 42, we considered the model of a beam.

355 A single simulation takes about 10-15 seconds of CPU time with a modern desktop computer. The computed deformed configurations of the structure for the time instant $t=10 \mathrm{~s}$ are presented in Fig. 5 . The curves here are simple plots of $u_{y}$ in dependence on the axial coordinate $x$, the domains of the finite elements are indicated by gray vertical lines, and the nodes of the finite element model reside on this lines. The effect of the bending stiffness is evident near the boundaries, and the difference because of the axial transport rate is also clear. More informative are the time histories of characteristic variables, which are shown for the three solutions in Fig. 6. In the middle point of the domain $x=l / 2$ we observe the time variation of the transverse displacement $u_{y}$, of 365 the strain $\varepsilon$ and of the axial component of the velocity of particles $v_{x}$; we also present the time variation of the strain $\varepsilon$ in the end point of the domain $x=l$. The following can be noticed.

- The strain at the right end immediately responds to the growing $y_{l}(t)$, but it takes some time before the influence in the middle point is observed.

- The starting values of the velocity $v_{x}$ are not equal to $\gamma$ because of the pre-strain, see Eq. (67).

- The bending stiffness makes the structure stiffer and slightly increases the natural frequency, which is most evident from the plot of $\left.u_{y}\right|_{x=l / 2}$. What is less intuitive is that the slower axial motion has a similar effect.

- The strain grows faster with the first kind of the boundary condition at the right end.

The last observation may seem to be contradictory, as the constant horizontal material velocity $v_{l}$ with higher $u_{x}^{\prime}$ means lower rate of material consumption, and the total material length of the string in the active domain is expected to grow. More detailed analysis shows, however, that in the first two seconds of the simulation we have growing $u_{y}^{\prime}$ and decreasing $u_{x}^{\prime}$ at the right end. This 

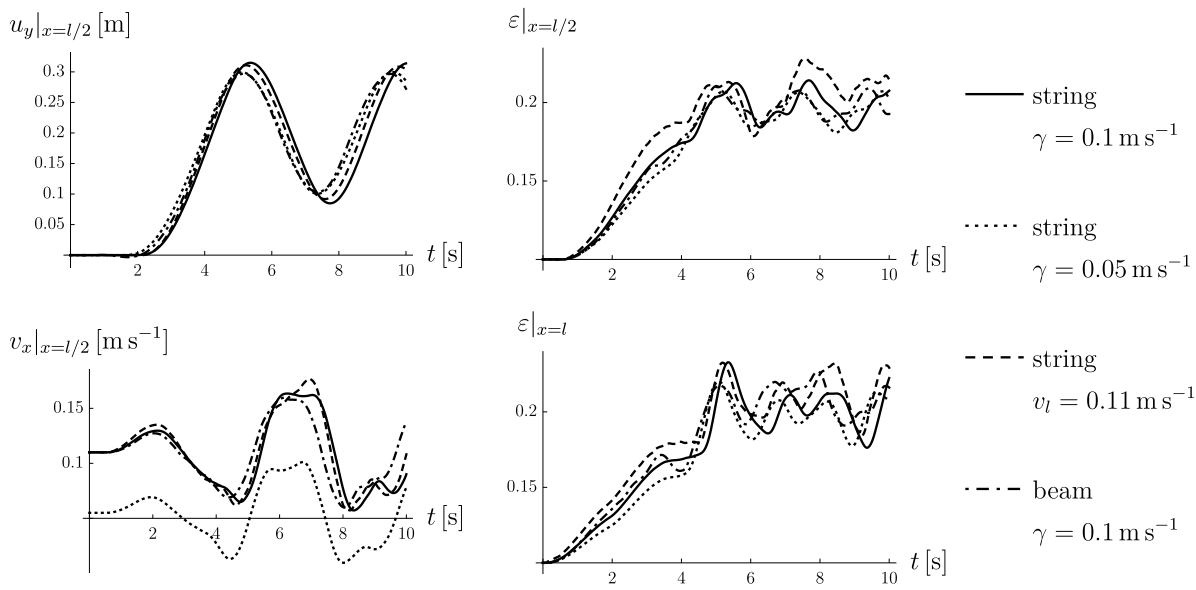

Figure 6: Time histories of the transverse deflection, strain and axial velocity in the middle point of the control domain as well as of the strain in the end point for the cases of a moving string, a string with twice slower axial transport, a string with the first type of boundary condition at the right end (horizontal material velocity $v_{l}$ prescribed instead of the rate of material consumption $\gamma$ ) and a beam

interplay of the components of $\mathbf{u}^{\prime}$ allows growing both $\varepsilon$ and the rate of material consumption while the material velocity remains constant. The situation would be different for a beam, which always has $u_{y}^{\prime}=0$ at $x=l$, and for which the first kind of the boundary condition results in lower $\varepsilon$ in the beginning of the simulation.

\subsection{Mesh convergence}

Convergence is probably the most important property of an applicable finite element scheme. Addressing this question, we considered the string model with the second kind of the boundary condition at the right end and sequentially increased the number of finite elements. Taking the solution with $n_{\mathrm{el}}=32$ as a reference one (and assuming it nearly converged), we plotted the absolute differences of the discussed characteristic variables between the solutions with $n_{\mathrm{el}}=4,8,16$ and the reference one in Fig. 7 in a logarithmic scale. The cubic finite element approximation provides a rapid mesh convergence and makes solutions with just a few finite elements accurate enough, at least for strings with no boundary effects.

\subsection{Verification against a Lagrangian lumped spring-mass model}

We decided to justify the converged solution against the results of a simulation with a lumped spring-mass model of the string, whose particles move across the domain. As with any discretized Lagrangian model, suppressing the numerical excitation of vibrations is the main challenge on this way: the particles periodically enter and leave the control domain, and we thus switch their motion law from the kinematically driven mode to the dynamic one. When the 

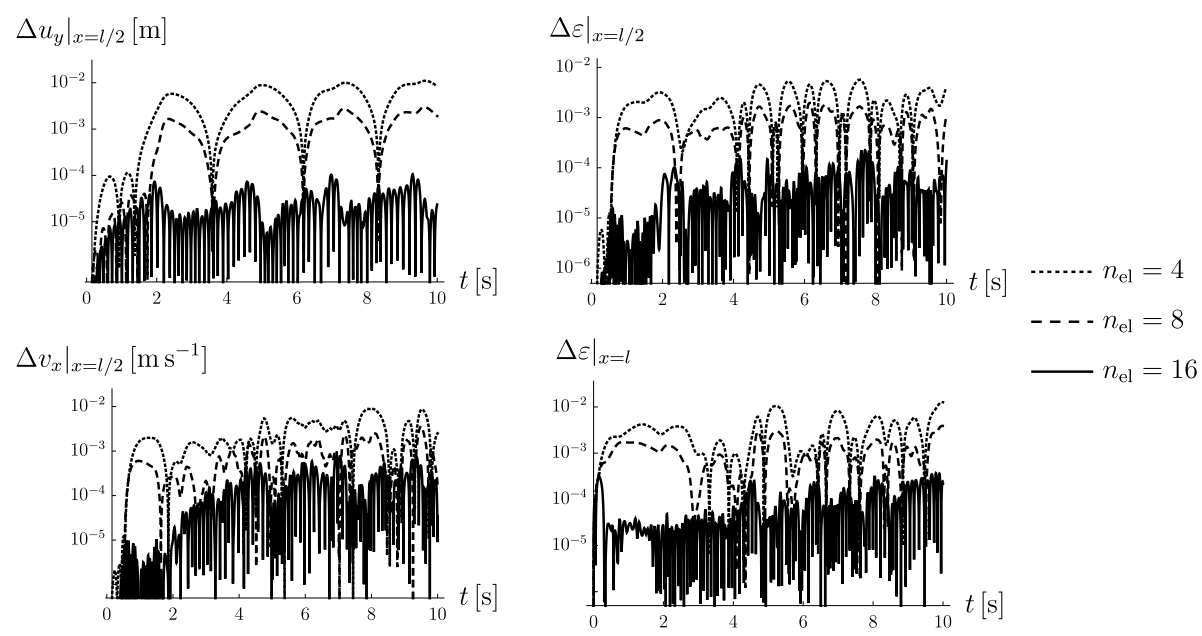

Figure 7: Time histories of absolute differences of characteristic variables from a converged solution for three levels of detail of the finite element mesh

velocity of the axial motion is comparable with the wave speed in the structure, the frequency of the numerical excitation lies in the densely populated range of the spectrum of natural frequencies of the numerical model, and the non-physical modes of vibration become excited. The effect is more prominent and difficult to suppress than in one-dimensional discrete particles simulations, discussed in the end of section 4.4. After several attempts to regularize the kinematic conditions in the domains $x<0$ and $x>l$ by submerging particles in artificial viscous flows with high viscosity factors, etc., we managed to obtain sufficiently smooth solutions adopting the strategy, which is briefly described below.

${ }_{415}$ Considering a system of $n_{\mathrm{sm}}$ masses, connected by elastic springs, we explicitly integrate the equations of dynamics over time with a sufficiently small time step size. The values of each mass, spring stiffness and undeformed spring length are such, that the undeformed length of the discrete structure, its total mass and stiffness equal respectively $s_{l}-s_{0}, \rho\left(s_{l}-s_{0}\right)$ and $b\left(s_{l}-s_{0}\right)^{-1}$ from the

420 continuous model. The first and the last particles are connected to the points $\mathbf{r}=0$ and $\mathbf{r}=l \mathbf{i}+y_{l}(t) \mathbf{j}$ by two springs, whose undeformed lengths and stiffness coefficients are varied between the time steps to represent the constant rate $\gamma$ of the influx of the material at $x=0$ and its outflux at $x=l$, which corresponds to the second kind of the boundary condition at the right end. As soon as the 425 undeformed length of the leftmost spring exceeds a certain threshold (which is by $50 \%$ larger than the length of the other springs in the model), we divide it into two by adding a new mass point at it. We also observe the undeformed length of the rightmost spring and remove the rightmost mass point as soon as it goes below $50 \%$ of the length of the other springs. Although this instantaneous ${ }_{430}$ insertion and removal of mass points during the time integration results in nu- 

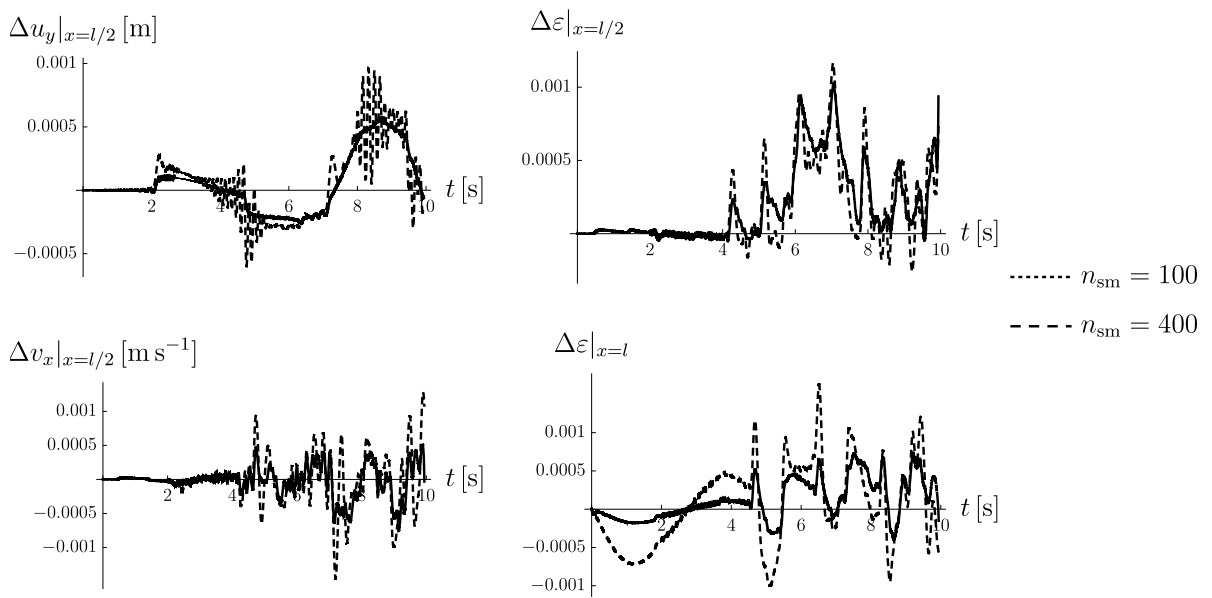

Figure 8: Time histories of differences of characteristic variables between the non-material finite element analysis and the Lagrangian discrete particles simulation with 100 and 400 material points

merically induced vibrations, the effect is much less pronounced in comparison to other tested modelling techniques.

In Fig. 8 we compare the above reference solution with $n_{\mathrm{el}}=32$ finite elements to the discrete particle simulations with $n_{\mathrm{sm}}=100$ and $n_{\mathrm{sm}}=400$

435 mass points. Computing the characteristic variables in the Lagrangian simulation, we had to switch the measurements to the particles, which are currently closest to the middle point of the domain. Along with vibrations, numerically induced by inserting and removing material points in the course of the time integration, this results in a small high-frequency component in the computed ${ }_{440}$ time histories, which we filtered during the post-processing stage in Wolfram Mathematica using a low-pass filter with a suitable cutoff frequency. Besides good correspondence of the results, obtained in the proposed non-material finite element model and the Lagrangian discrete particles simulation, we also observe the convergence of the results as the number of particles is increasing ${ }_{445}$ (besides $\left.\varepsilon\right|_{x=l / 2}$, which is yet to be understood). The accuracy of the model with $n_{\mathrm{sm}}=400$ particles in on the same level with the simulation, which features $n_{\mathrm{el}}=16$ finite elements. The success of this comparison leaves little space for doubts regarding the correctness of the above theoretical considerations and the transformation of the material formulation of D'Alembert's principle of virtual work to the Lagrange's equations of motion of the second kind, written for the non-material control volume.

\subsection{Verification against a Lagrangian beam model}

The second option to validate the presented mixed Eulerian-Lagrangian kinematic description against a conventional Lagrangian model is to consider a

beam, which is not moving across the domain by setting $\gamma=0$. The entire 


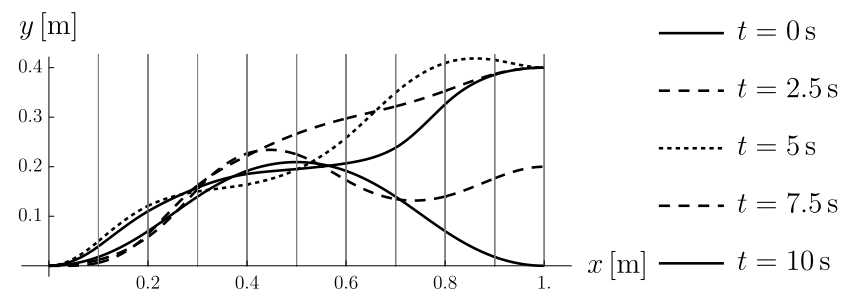

Figure 9: Intermediate configurations of an initially buckled beam with the moving right end

material volume is thus preserved, but the particles still move across the boundaries of the non-material finite elements. As the beam is not moving axially, we may now use both the Eulerian and the conventional Lagrangian finite element models and compare the results. The two numerical schemes are not equivalent, but are expected to converge to the same solutions when the mesh is refined.

The effect of the bending stiffness is more pronounced in a setting, in which the beam is initially compressed and takes an equilibrium shape after the stability is lost. Consider a beam of the material length $s_{l}-s_{0}=l /\left(1-\varepsilon_{0}\right)$, whose end points are clamped horizontally in $\mathbf{r}=0$ and $\mathbf{r}=l \mathbf{i}$. A straight configuration 465 would have $\varepsilon=-\varepsilon_{0}$ and becomes unstable for the chosen value of pre-strain Eq. (65). The analysis features thus the following two steps.

1. Using the finite element model and an imperfect initial configuration, we find a stable static equilibrium, which provides a curved supercritical state with $u_{y}>0$.

2. Considering the equilibrium state as an initial one, we solve the transient dynamic problem, in which the structure is brought into motion by timevarying transverse coordinate of the right end $y_{l}(t)$.

The presence of the two states of equilibrium, which are getting closer to each other as the right end is moved and $y_{l}>0$, enriches the dynamics of the system with highly nonlinear effects. Dynamical configurations of the beam at five sequential time instances are demonstrated in Fig. 9 for the model with $n_{\mathrm{el}}=10$ finite elements. Time histories of the same characteristic variables as before are plotted for the problem at hand in Fig. 10 for three levels of mesh detail. Evidently, the mesh with $n_{\mathrm{el}}=4$ is too coarse for a beam problem, in which 480 boundary effects play an essential role, but the other two solutions are close to each other. Further mesh refinement shows that the solution with $n_{\mathrm{el}}=16$ is nearly converged.

We solved the same problem in a conventional Lagrangian formulation with the same cubic approximation for the beam finite elements [20, 37, 40. Among the earlier treated characteristic variables we choose the strain at the right end $\left.\varepsilon\right|_{x=l}$ for comparison because of three reasons. First, this variable is easier to retrieve in the Lagrangian simulation, as the material point at $x=l$ is always the same. Second, the finite element solution for a beam model converges here slower than in the middle because of the boundary effect, which makes this 

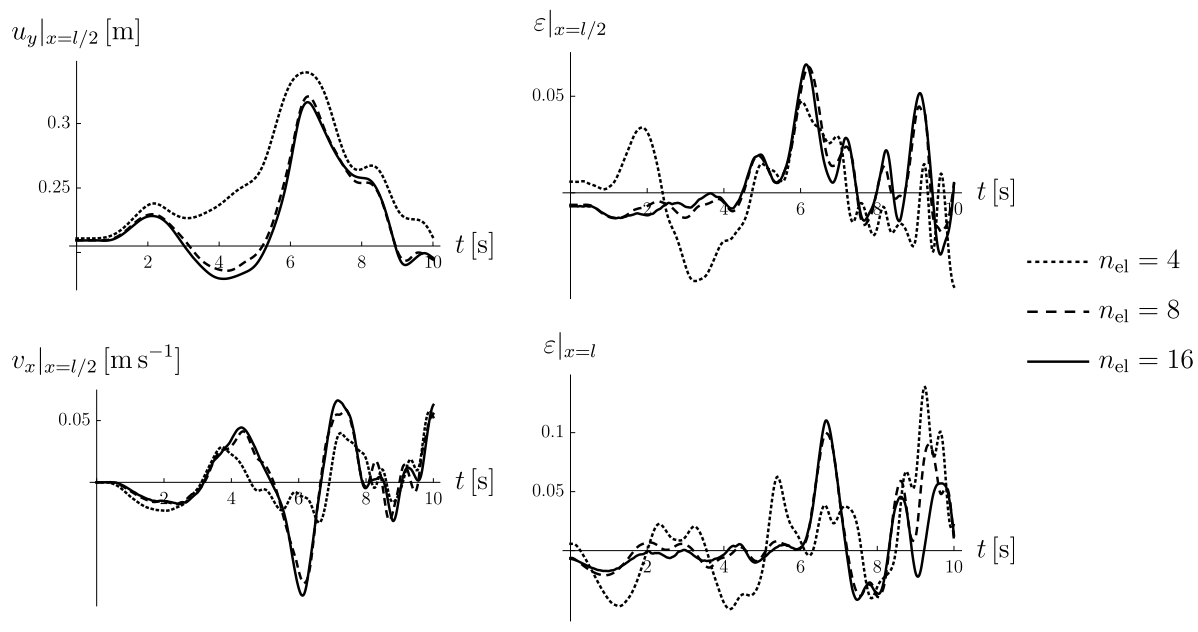

Figure 10: Characteristic variables in the dynamics problem of an initially buckled beam for the three levels of detail of the finite element mesh

490 overwhelm the reader with too many plots. Considering the solution with the above model and 32 finite elements as a reference one, in Fig. 11 we logarithmically plotted the absolute differences of the solution with 16 finite elements as well as of the solutions with 32 and 16 finite elements, obtained with the fully Lagrangian model. Both coarser models produce the same level of accuracy, and the solutions with finer meshes are remarkably close to each other. Not aiming at obtaining the perfectly converged solution, this way we demonstrate the mutual convergence of the mixed Eulerian-Lagrangian model and the fully Lagrangian one.

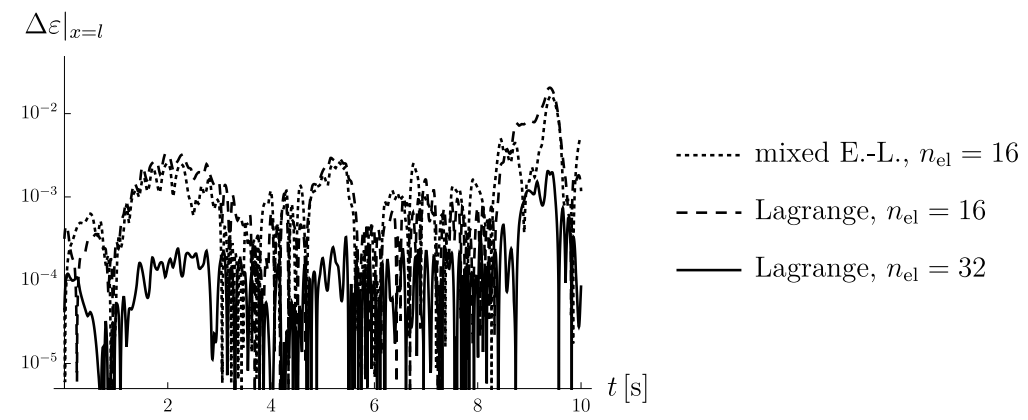

Figure 11: Time histories of the absolute difference of the strain at the right end between the reference solution and the mixed Eulerian-Lagrangian model with 16 finite elements, Lagrangian model with 16 finite elements, Lagrangian model with 32 finite elements 

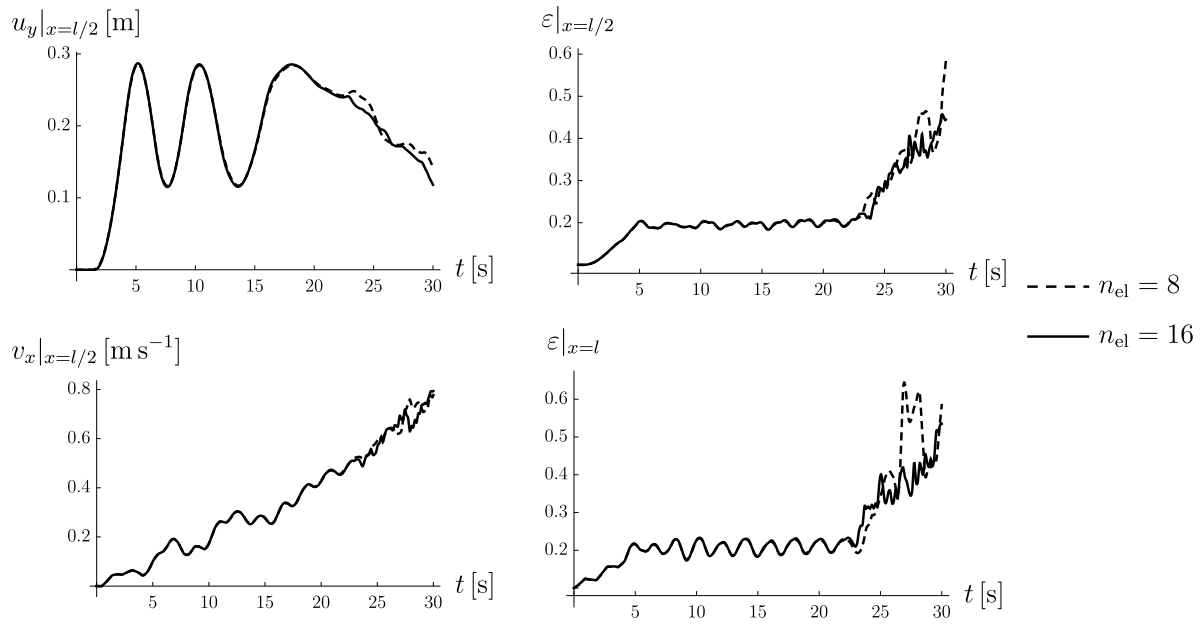

Figure 12: Dynamic instability: time histories of characteristic variables at accelerated motion, computed with two finite element discretizations

\subsection{Accelerated motion and dynamic instability}

As we already have mentioned in the end of section 2 , steady-state solutions become unstable as the rate of axial transport exceeds some critical value. Linearly increasing $\gamma$ from 0 to 0.55 over the time span from $t=0$ to $t=30$, we modelled the accelerated motion of a pre-tensed string with 8 and 16 finite elements, see Fig. 12. Nothing specific happens until $t=20$ besides the slight increase in the period of oscillations of the middle point of the string, which indicates "softening" of the structure. But at some point, the solution "explodes" into a kind of chaotic behavior with very high strains. No mesh convergence could be observed in this super-critical state, which is no surprise as the partial differential equations governing the dynamics of the systems have changed their type. Particular danger of this kind of instability for technical applications increases the value of the presented theoretical results.

Recalling the theoretical outcome of the linear analysis in section 2, we may now estimate the value of the critical velocity by computing the wave speed $c$ of a stationary straight string, stretched between the two points. The material length of the string is $s_{l}-s_{0}=l /\left(1+\varepsilon_{0}\right) \approx 0.909 \mathrm{~m}$, its geometrical length is $l^{*}=\sqrt{l^{2}+\left(y_{l}^{*}\right)^{2}} \approx 1.077$, which means the stationary strain $\varepsilon^{*}=$ $l^{*} /\left(s_{l}-s_{0}\right)-1 \approx 0.185$. With the tension force $T^{*}=b \varepsilon^{*}$ and the actual linear density $\rho^{*}=\rho\left(s_{l}-s_{0}\right) / l^{*} \approx 0.844 \mathrm{~kg} \mathrm{~m}^{-1}$ we compute the speed of the transverse waves in the string

$$
c=\sqrt{T^{*} / \rho^{*}} \approx 0.468 \mathrm{~m} \mathrm{~s}^{-1} .
$$

From the one-dimensional relations Eq. (6) and Eq. (7) follows

$$
v=\dot{u}(1+\varepsilon) .
$$


Setting now $\dot{u}=\gamma$ for the stationary straight motion, we find that the velocity of the string gets equal to the wave speed at $\gamma=c /\left(1+\varepsilon^{*}\right) \approx 0.395 \mathrm{~m} \mathrm{~s}^{-1}$. This rate

515 of material supply is achieved at the time instant $t \approx 21.54 \mathrm{~s}$, which can indeed be identified as a starting point of the unstable behavior in Fig. 12. Addressing again the linear model Eq. (3), we confirm the conclusion that the wave motion is possible only as long as the velocity of the string is below the wave speed. The supercritical range appears to be inappropriate for the presented non-material

520 finite element analysis. As a possible explanation we may guess that some of the required continuity conditions are no longer fulfilled.

\section{Conclusions}

We have applied the mixed Eulerian-Lagrangian kinematic description to the dynamics of axially moving strings and beams using D'Alembert's form of ${ }_{525}$ the equation of virtual work. The equation is rewritten for a spatially fixed range of integration with the possibility to introduce approximations of the unknown displacements over the considered control domain. Assuming kinematic boundary conditions, we managed to transform the variational equality for a discretized system to the form of Lagrange's equations of motion of the second kind, which allows for a particularly simple numerical implementation. The resulting finite element scheme has been validated against the results of a discrete particle simulation and another fully Lagrangian finite element model. We have also demonstrated rapid mesh convergence as well as the ability of the model to represent important effects like softening of the structure with increasing axial 535 velocity and dynamic instability. The mathematical model is relevant for engineering applications and allows for an extension towards finite element analysis of nonlinear dynamics of axially moving plates.

\section{Acknowledgment}

The author expresses his deep gratitude to his former and present colleagues 540 Prof. Hans Irschik from the Johannes Kepler University Linz, member of Austrian and Russian academies of sciences; Prof. Vladimir Eliseev from the Peter the Great St. Petersburg Polytechnical University; Prof. Michael Krommer from the Vienna University of Technology. Their attention to the present research and ongoing discussions played a crucial role in the emergence of the paper.

545 The support of the author from the Austrian Science foundation FWF in the framework of the collaboration with the research team of the project "Eulerian Mechanics of Belts" [grant number I2093-N25] is gratefully acknowledged.

\section{References}

[1] L.-Q. Chen, Analysis and Control of Transverse Vibrations of Axially Moving Strings, ASME Applied Mechanics Reviews 58 (2005) 91-116. 
[2] K. Marynowski, T. Kapitaniak, Dynamics of axially moving continua, International Journal of Mechanical Sciences 81 (2014) 26-41.

[3] M. H. Ghayesh, M. Amabili, M. P. Païdoussis, Nonlinear dynamics of axially moving plates, Journal of Sound and Vibration 332 (2) (2013) 391-406.

[4] L. Kong, R. Parker, Steady mechanics of belt-pulley systems, ASME Journal of Applied Mechanics 72 (2005) 25-34.

[5] J. Wickert, Nonlinear vibration of a traveling tensioned beam, International Journal of Non-Linear Mechanics 27 (3) (1992) 503-517.

[6] J. Mote, On the nonlinear oscillation of an axially moving string, Journal of Applied Mechanics 33 (1966) 463-464.

[7] L. Kong, R. Parker, Vibration of an axially moving beam wrapping on fixed pulleys, Journal of Sound and Vibration 280 (2005) 1066-1074.

[8] F. Pellicano, G. Catellani, A. Fregolent, Parametric instability of belts: theory and experiments, Computers and Structures 82 (2004) 81-91.

565 [9] F. Pellicano, On the dynamic properties of axially moving systems, Journal of Sound and Vibration 281 (2005) 593-609.

[10] X.-D. Yang, H. Wu, Y.-J. Qian, W. Zhang, C. Lim, Nonlinear vibration analysis of axially moving strings based on gyroscopic modes decoupling, Journal of Sound and Vibration In Press.

[11] A. Humer, Dynamic modeling of beams with non-material, deformationdependent boundary conditions, Journal of Sound and Vibration 332 (2013) 622-641.

[12] A. Humer, H. Irschik, Onset of transient vibrations of axially moving beams with large displacements, finite deformations and an initially unknown length of the reference configuration, ZAMM Zeitschrift fur Angewandte Mathematik und Mechanik 89 (4) (2009) 267-278.

[13] A. Humer, H. Irschik, Large deformation and stability of an extensible elastica with an unknown length, International Journal of Solids and Structures 48 (2011) 1301-1310.

[14] V. Eliseev, Y. Vetyukov, Effects of deformation in the dynamics of belt drive, Acta Mechanica 223 (2012) 1657-1667.

[15] A. Pechstein, J. Gerstmayr, A Lagrange-Eulerian formulation of an axially moving beam based on the absolute nodal coordinate formulation, Multibody System Dynamics 30 (2013) 343-358.

[16] Y. Vetyukov, E. Oborin, M. Krommer, V. Eliseev, Transient modelling of flexible belt drive dynamics using the equations of a deformable string with discontinuities, Mathematical and Computer Modelling of Dynamical Systems online first (2016) 1-15. 
[17] J. Donea, A. Huerta, J.-P. Ponthot, A. Rodriguez-Ferran, Arbitrary Lagrangian-Eulerian Methods, in: E. Stein, R. de Borst, T. Hughes (Eds.), Encyclopedia of Computational Mechanics, Vol. 1: Fundamentals, John Wiley \& Sons, Ltd, 2004, Ch. 14.

[18] D. Hong, G. Ren, A modeling of sliding joint on one-dimensional flexible medium, Multibody System Dynamics 26 (2011) 91-106.

[19] S. Yang, Z. Deng, J. Sun, Y. Zhao, S. Jiang, A variable-length beam element incorporating the effects of spinning, Latin American Journal of Solids and Structures (in press).

[20] Y. Vetyukov, Nonlinear Mechanics of Thin-Walled Structures. Asymptotics, Direct Approach and Numerical Analysis, Foundations of Engineering Mechanics, Springer, Vienna, 2014.

[21] H. Irschik, M. Krommer, M. Nader, Y. Vetyukov, H.-G. von Garssen, The equations of Lagrange for a continuous deformable body with rigid body degrees of freedom, written in a momentum based formulation, Journal of Sound and Vibration 335 (2015) 269-285.

[22] D. McIver, Hamilton's principle for systems of changing mass, Journal of Engineering Mathematics 7 (3) (1973) 249-261.

[23] U. Zwiers, On the Dynamics of Axially Moving Strings, Cuvillier, Göttingen, 2007.

[24] D. Kim, M. Leamy, A. Ferri, Dynamic Modeling and Stability Analysis of Flat Belt Drives Using an Elastic/Perfectly Plastic Friction Law, ASME Journal of Dynamic Systems, Measurement, and Control 133 (2011) 1-10.

[25] H. Hetzler, On moving continua with contacts and sliding friction: Modeling, general properties and examples, International Journal of Solids and Structures 46 (2009) 2556-2570.

[26] M. Stangl, J. Gerstmayr, H. Irschik, An alternative approach for the analysis of nonlinear vibrations of pipes conveying fluid, Journal of Sound and Vibration 310 (3) (2008) 493-511.

[27] M. Païdoussis, Fluid-Structure Interactions: Slender Structures and Axial Flow, 2nd Edition, Vol. 1, Elsevier, 2013.

[28] H. Irschik, H. Holl, The equations of Lagrange written for a non-material volume, Acta Mechanica 153 (2002) 231-248.

[29] H. Irschik, H. Holl, Lagrange's equations for open systems, derived via the method of fictitious particles, and written in the Lagrange description of continuum mechanics, Acta Mechanica 226 (2015) 63-79. 
[34] V. Eliseev, Mechanics of deformable solid bodies (in Russian), St.Petersburg State Polytechnical University Publishing House, St. Petersburg, 2006.

[35] A. Lurie, Analytical Mechanics, Springer, Berlin, 2002.

640

[30] Y. Vetyukov, P. Gruber, M. Krommer, Nonlinear model of an axially moving plate in a mixed Eulerian-Largangian framework, Acta Mechanica 227 (2016) 2831-2842.

[31] Y. Vetyukov, P. Gruber, M. Krommer, J. Gerstmayr, I. Gafur, G. Winter, Mixed Eulerian-Lagrangian description in materials processing: deformation of a metal sheet in a rolling mill, International Journal for Numerical Methods in Engineering online first (2016) 1-20.

[32] W. van Horssen, S. Ponomareva, On the construction of the solution of an equation describing an axially moving string, Journal of Sound and Vibration 287 (1-2) (2005) 359-366.

[33] K. Graff, Wave motion in elastic solids, Clarendon Press, Oxford, 1975.

[36] F. Gantmakher, Lectures in Analytical Mechanics, Mir Publishers, Moscow, 1970.

[37] Y. Vetyukov, Hybrid asymptotic-direct approach to the problem of finite vibrations of a curved layered strip, Acta Mechanica 223 (2) (2012) 371385.

${ }_{645}[38]$ S. Antman, The theory of rods, in: S. Flügge, C. Truesdell (Eds.), Handbuch der Physik, Vol. VIa/2, Springer, Berlin-Heidelberg-New York, 1972, pp. 641-703.

[39] Y. Vetyukov, Finite element modeling of Kirchhoff-Love shells as smooth material surfaces, ZAMM 94 (1-2) (2014) 150-163.

[40] P. Gruber, K. Nachbagauer, Y. Vetyukov, J. Gerstmayr, A novel directorbased Bernoulli-Euler beam finite element in absolute nodal coordinate formulation free of geometric singularities, Mechanical Sciences 4 (2013) 279-289. 